\title{
Feast and famine in agricultural research
}

\author{
Ending hunger is a major objective of the United Nations' Sustainable Development Goals. A cross-journal \\ collection of articles takes a systematic look at what we might already know about achieving it.
}

T

he global health emergency is both overshadowing and exacerbating chronic problems that existed well before this time last year. World Food Day, which falls on 16 October 2020, would have been important enough had its focus only been the United Nations' second Sustainable Development Goal to abolish hunger by 2030. But according to the World Food Program's 2020 Global Report on Food Crises $^{1}$, the last year has seen at least 130 million more people at risk of hunger as a result of COVID-19. Countless others have discovered that food security is not permanent, and that the fall from food abundance to food scarcity can occur in a matter of weeks when one's access to income is upended ${ }^{2}$. One credible estimate of the additional investment needed to prevent millions more people from becoming food insecure as a result of COVID-19 is US\$10 billion ${ }^{3}$.

This month, a collection (https://www. nature.com/collections/dhiggjeagd) of articles published across a number of Nature Research journals (Nature Food, Nature Machine Intelligence, Nature Plants and Nature Sustainability) attempts to tackle this problem head on. In so doing it starkly highlights that the scarcity and abundance endemic in our actual food systems is mirrored in the body of research about agriculture and food systems. Despite a systematic scouring of the literature aided by sophisticated machine-learning approaches, scant research can be found concentrating on one of the largest populations of hungry people: small-scale farmers.

These studies emerge from the Ceres2030: Sustainable Solutions to End Hunger project, which brought together more than 75 global experts from 23 countries. These researchers looked at a diverse set of issues in their evaluation of more than 100,000 articles in agricultural research. Using detailed protocols registered on the Open Science Framework (https:// osf.io/adxek/) before the work commenced, they identified all articles capable of contributing to their scientific assessment. A surprisingly consistent result was that only around $2 \%$ of published agricultural and agronomic research has original and high-quality data about solutions for small-scale producers ${ }^{4}$.

However, their scientific assessment should be a clear reminder that $2 \%$ of an evidence base is not zero. Despite the patchy nature of the evidence base, the teams have identified many robust and significant effects.

- Farmers are increasingly under pressure to switch to new, climate-resilient crops due to uncertain and constantly changing weather patterns. Higher rates of crop adoption by farmers are linked to training and advisory services.

- In low- and middle-income countries, $76.7 \%$ of small-scale farms are located in water-scarce regions, and fewer than $37.2 \%$ of these have irrigation systems. These water needs must be addressed, and the use of livestock and digital technologies are under-assessed alternatives to irrigation.

- Farmers' organizations are particularly helpful to smallholder farmers. Membership was associated with positive effects on income in $57 \%$ of the cases reviewed, and on crop yield, on production and on the environment in $20-25 \%$ of studies.

There are also uncomfortable data on the degree to which women - representing nearly $50 \%$ of the agricultural workforce in the countries studied ${ }^{5}-$ are overlooked when assessing the efficacy of aid initiatives, with only around one in ten studies considering the outcomes for different genders. The costing of interventions is another factor frequently not included despite this being a vital piece of information to support policymaking.

Two Perspectives in the collection point out that the questions asked by policymakers and by researchers are different, and each is frustrated by the other's time scales ${ }^{4,6}$. But two things both parties agree on are a lack of evidence in the evidence base, and that researchers are forced to waste an inordinate amount of time due to inefficiencies in the research process ${ }^{7}$.
Sustainable Development Goal 2 is a constant reminder that we need to transform food systems to deliver affordable, nutritious and sustainable diets for all. Even before the COVID-19 pandemic the clock was ticking, and millions of people were going hungry every day ${ }^{8}$. When faced with a highly variable body of research capable of guiding our responses to such threats, it is imperative to employ every possible byte of data. Yet in most disciplines - agriculture included - the methodological processes and standards needed to help analyse the results of thousands of heterogeneous studies are still in their infancy?.

Agriculture is faced with a very broad array of information sources and study designs, hampering the effectiveness of many evidence synthesis approaches ${ }^{10}$. The researchers of the Ceres2030 project must be applauded for demonstrating to the full the powers of evidence syntheses - especially the frequently undervalued Scoping Review - to mine even the messiest of evidence bases.

The ability to engage in informed, systematic and rational decision making will be essential in tackling hunger and poverty in general. This is not only necessary and urgent but, as Ceres2030 has demonstrated, also achievable.

Published online: 13 October 2020 https://doi.org/10.1038/s41477-020-00795-9

References

1. 2020 Global Report on Food Crises (Food Security Information Network, 2020).

2. Goodman, P. S., Dahir, A. L. \& Singh, K. D. The Other Way Covid Will Kill: Hunger. The New York Times (11 September 2020).

3. Laborde, D. \& Smaller, C. What Would it Cost to Avert the COVID-19 Hunger Crisis? (Ceres2030, 2020).

4. Porciello, J., Ivanina, M., Islam, M., Einarson, S. \& Hirsh, H. Nat. Mach. Intell. https://doi.org/10.1038/s42256-020-00235-5 (2020).

5. The State of Food and Agriculture 2010-11, Women in Agriculture: Closing the Gender Gap for Development. Food and Agriculture Organization of the United Nations http://www.fao. org/3/i2050e/i2050e.pdf (2011).

6. Lipper, L., DeFries, R. \& Bizikova, L. Nat. Plants https://doi. org/10.1038/s41477-020-00792-y (2020).

7. Chalmers, I. et al. Lancet 383, 156-165 (2014).

8. The State of Food Security and Nutrition in the World 2020: Transforming Food Systems for Affordable Healthy Diets (FAO, IFAD, UNICEF, WFP and WHO, 2020).

9. Gurevitch, J., Koricheva, J., Nakagawa, S. \& Stewart, G. Nature 555, 175-182 (2018).

10. Webb, P. \& Kennedy, E. Food Nutr. Bull. 35, 126-132 (2014). 